\title{
La falta de acceso a la interrupción voluntaria del embarazo trae consecuencias psicológicas negativas en las mujeres
}

Lack of access to voluntary termination of pregnancy abortion leads to negative psychological consequences in women

\section{Objetivos}

Evaluar el bienestar psicológico de las mujeres luego de haber accedido o de habérseles rechazado la posibilidad de realizar una interrupción voluntaria del embarazo (IVE) durante un seguimiento de cinco años.

\section{Diseño, lugar y pacientes}

Estudio longitudinal prospectivo, realizado en treinta centros que realizan interrupción legal del embarazo en 21 estados de EE.UU., con reclutamiento entre 2008 y 2010 , y seguimiento hasta 2016 . El umbral o edad gestacional límite para la finalización del embarazo podía ser diferente en cada centro, con rango variable desde 10 semanas hasta el final del segundo trimestre. Las pacientes debian tener al menos 15 años de edad y presentar embarazos viables, sin malformaciones o anomalías conocidas que implicaran riesgos para la salud física de la mujer. Las participantes fueron incluidas en tres grupos (proporción 2:1:1): a) mujeres con edad gestacional dentro de las dos semanas de la fecha umbral límite que accedieron a la IVE (grupo peri-umbral, $n=452$ ); $b$ ) mujeres cursando embarazo dentro del primer trimestre que accedieron a esta práctica (grupo primer trimestre, $n=273$ ); y c) mujeres cursando embarazo dentro de las tres semanas de excedido el umbral a quienes se les rechazó esta práctica (grupo post-umbral, $\mathrm{n}=231$ ). Este último grupo además se subdividió en aquellas mujeres que dieron a luz (postumbral nacidos, $n=161$ ) y aquellas que igualmente lograron realizarse un aborto por otros medios (post-umbral no nacidos, $n=70$ ).

\section{Medición de resultados principales}

Se realizaron mediciones de depresión y ansiedad (subescalas de depresión y ansiedad del Inventario Breve de Síntomas, en inglés: Brief Symptom Inventory), de autoestima y de satisfacción auto-percibidas. Estos desenlaces se midieron mediante entrevistas telefónicas a los ocho días de la evaluación de la práctica o del rechazo del aborto, y luego semestralmente, durante cinco años. Las variables de
Biggs MA y col. JAMA Psychiatry 2017;74(2):169-178 ajuste incluyeron: edad, origen étnico auto-reportado, nivel educativo más alto alcanzado, estado civil, empleo, paridad, antecedentes de abuso o negligencia infantil, antecedentes de depresión o ansiedad, y consumo de drogas ilícitas y de alcohol antes del embarazo.

\section{Resultados principales}

De las 956 mujeres incluidas en el estudio (edad media: 24,9 años; desvío estándar: 5,8), una semana después de la búsqueda de una IVE, las mujeres a las cuales se les había negado esta práctica reportaron más síntomas de ansiedad, menor autoestima y menor satisfacción. La ansiedad disminuyó con el tiempo, excepto en aquellas mujeres que no accedieron a la IVE por otros medios y dieron a luz. La autoestima y la satisfacción mejoró en todos los grupos con el pasar del tiempo. Hubo mayores síntomas depresivos en aquellas mujeres a quienes se les negó la práctica del aborto, y mayor número de casos de depresión en el grupo que este procedimiento no se logró por otros medios. Para todos los grupos, los síntomas de depresión mejoraron con el tiempo. Todos los resultados fueron similares entre los grupos a largo plazo.

\section{Conclusiones}

En este estudio, la negación del acceso a la práctica de un aborto o IVE se asoció con un mayor riesgo de experimentar resultados psicológicos adversos a corto plazo. El bienestar psicológico mejoró con el tiempo y no se mostraron diferencias entre los grupos a largo plazo. Estos hallazgos sugieren que no sería necesario advertir a las mujeres sobre las consecuencias psicológicas negativas de realizarse este procedimiento.

Fuente de financiamiento/conflictos de interés de los autores: No se reportan conflictos de interés. Este estudio fue financiado por Wallace Alexander Gerbode Foundation, David and Lucile Packard Foundation, The William and Flora Hewlett Foundation y un donante anónimo. Ninguno de los financiadores tuvo roles en el diseño, conducción o el reporte del estudio.

\section{Comentario}

El aborto ha sido manipulado por distintas disciplinas tales como la medicina, la psicología, la abogacía o la religión, por citar tan solo algunas de ellas. Todos intentan dar una mirada objetiva sobre el tema, pero en realidad todos dejan la rigurosidad del pensamiento y del conocimiento para adentrarse en las inconsistentes aguas de la política. Quienes se oponen al aborto han intentado con poco éxito calificarlo como una intervención que deja graves secuelas emocionales en la vida de la mujer (Sindrome post aborto o SPA, en inglés: Post Abortion Syndrome). Ya la Asociación Americana de Psicología (en inglés: American Psychological Association) en 2008 había mencionado que el SPA tenía poca evidencia científica, y que en todo caso era la percepción de su entorno la que producía efectos nocivos emocionales en las mujeres ${ }^{1}$. A pesar del poco sustento científico, son varios los estados de EE.UU. que han generado, sobre la base del SPA, legislaciones restrictivas para el logro de la interrupción de un embarazo. Se obliga a las mujeres a ver el feto en una ecografía, o a escuchar los latidos, o a esperar 72 horas antes de una estrategia de interrupción, o se ven sometidas a un tribunal o comité de bioética con la intención de disuadirlas. Asimismo, estas legislaciones o actitudes intentan mostrar que la mujer toma este tipo de decisiones sobre bases poco sólidas. En este estudio resumido, el grupo liderado por la Dra. Diana Foster, demógrafa, viendo que existían pocos datos objetivos al respecto, se preguntó cuáles eran las consecuencias de un embarazo no deseado, o cuál era el grado de veracidad del SPA. Para ello siguió a un grupo de casi 1.000 mujeres durante un período de 5 años, dividiendo los grupos entre quienes habían tenido un aborto en

tiempo y forma, y las que habían tenido que seguir con un embarazo por no haber logrado un aborto, dado que se habían excedido en el tiempo legal. El análisis se centró en los aspectos emocionales de las mujeres que habían tenido un aborto, o si el nuevo niño producto de estos embarazos, aumentaba las dificultades sociales o financieras de la mujer. La cantidad de entrevistas telefónicas al final del estudio fueron aproximadamente 8.000. El estudio mostró que las mujeres que habían tenido que seguir con un embarazo al término, tenían peores resultados que las que habían logrado un aborto en tiempo y forma. Estudios previos habían documentado cuáles eran las razones por las que las mujeres accedían a un aborto y la relevancia de sus decisiones. El $60 \%$ de las mujeres que acceden a un aborto ya han tenido un hijo previamente' por lo que la mayoría de las mujeres ya sabe lo que es la maternidad y cuáles son sus costos. De la misma manera se ha demostrado que las mujeres interrumpen un embarazo no deseado por preocupación o responsabilidad por otros $(74 \%)$, por no poder mantener económicamente un hijo o un nuevo hijo (73\%), porque un nuevo hijo puede interferir con su educación o su trabajo (69\%), por ser una mujer soltera y sin pareja $(48 \%)$ o por haber completado su tamaño familiar (38\%).

\section{Conclusiones del comentador}

El trabajo de Antonia Biggs y col. muestra al día de hoy la inconsistencia del SPA. Por ello es fundamental que la medicina a través de cada uno de los componentes del equipo de salud y sus sociedades científicas vean la necesidad que el aborto sea un bien social y, como tal, legal y seguro.

Mario Sebastiani [ Servicio de Obstetricia y Comité de Bioética Asistencial. Hospital Italiano de Buenos Aires. mario.sebastiani@hospitalitaliano.org.ar ] Sebastiani M. La falta de acceso a la interrupción voluntaria del embarazo trae consecuencias psicológicas negativas en las mujeres. Evid Act Pract Ambul 2018;21(2):58. Comentado de: Biggs MA y col. Women's Mental Health and Well-being 5 Years After Receiving or Being Denied an Abortion: A Prospective, Longitudinal Cohort Study. JAMA Psychiatry 2017;74(2):169-178

\section{Referencias}

1. J American Psychological Association. APA Task Force Finds Single Abortion Not a Threat to Women's Mental Health. Disponible en: http://www.apa.org/news/press/ releases/2008/08/single-abortion.aspx (último acceso 27 de junio de 2018). 2.Finer LB y col. Reasons U.S. women have abortions: quantitative and qualitative perspectives, Perspectives on Sexual and Reproductive Health, 2005, 37(3):110-118. 